\title{
Miniaturization of Quasi-Yagi Antenna Array with High Gain Using Split-Ring Resonators
}

\author{
Wenying Zhou (iD and Mai Lu (iD \\ Key Laboratory of Opto-Technology and Intelligent Control Ministry of Education, Lanzhou Jiaotong University, Lanzhou, \\ Gansu 730070, China \\ Correspondence should be addressed to Mai Lu; mai.lu@hotmail.com
}

Received 1 February 2020; Revised 7 April 2020; Accepted 13 April 2020; Published 22 May 2020

Academic Editor: Chien-Jen Wang

Copyright (C) 2020 Wenying Zhou and Mai Lu. This is an open access article distributed under the Creative Commons Attribution License, which permits unrestricted use, distribution, and reproduction in any medium, provided the original work is properly cited.

\begin{abstract}
In order to realize the miniaturization of quasi-Yagi antenna array, an antenna array with split-ring resonators (SRRs) based on two 7 units quasi-Yagi elements is designed in this paper. The radiation performance of the quasi-Yagi antenna array degrades significantly when array element spacing is reduced. After embedding SRRs on both sides of the miniaturized dielectric substrate surface, the $S$ parameters and gain of array are significantly better than the array without SRRs, and the adjustable wave beam energy is also enhanced effectively. It indicates that the proposed antenna array with SRRs has good directional radiation performance under the miniaturize process at the operation frequency of $2.45 \mathrm{GHz}$, which could be widely applied in the fields of smart rail transportation and wireless power transfer.
\end{abstract}

\section{Introduction}

With the development of the intelligent transportation networks and the new energy Internet of Things (IOT), the antenna array for transmitting wireless communication signals needs to achieve miniaturized appearance and high gain radiation. The quasi-Yagi antenna is a typical representative of end-fire antennas. Due to good directionality and high gain, the quasi-Yagi antenna was most widely used in wireless communication as vehicle-mounted antenna or access points (AP) in metro tunnels [1-3]. In order to overcome the frequency selective fading caused by the multipath effect, the stacked quasi-Yagi array for $2.45 \mathrm{GHz}$ has been used in wireless charging systems of REID chips [4]. The quasi-Yagi antenna by using the metamaterial concept has been utilized in wireless power transfer (WPT) to medical implants [5]. The beam-forming was also very important for multi-input-output (MIMO) communication [6]. A quasi-Yagi array of four radiating elements [7] and a wideband sectoral quasi-Yagi antenna has been presented for the MIMO system [8]. The mutual coupling [9] effect of quasi-Yagi antenna arrays was a ubiquitous problem in wireless communication [10]. The large reflection of the radiation port could cause the pattern distortion and gain reduction [11]. Therefore, the research on decoupling of the quasi-Yagi antenna array has practical application significance.

The electromagnetic band gap (EBG) $[12,13]$, defected ground structure (DGS) $[14,15]$, and metamaterials $[16,17]$ could be applied to suppress mutual coupling of antennas. As the reflector of quasi-Yagi has been printed on the back of the dielectric substrate, the high-surface required for the EBG structure and the DGS gap could not be inscribed, which would affect the induction field on the reflector. Due to the feature of suppressing electromagnetic energy transmission, the single-negative magnetic metamaterials have been widely used to reduce mutual coupling between high-profile antennas [18-20] and have adopted different SRRs structures [21-23], but few were used for quasi-Yagi antenna arrays.

The proposed antenna array based on two 7-units quasiYagi antenna elements adopt the single-negative magnetic metamaterial concept to suppress the mutual coupling of elements, and its different balun compound mode could 
form an adjustable beam for directional radiation through embedding the SRRs on both sides of the dielectric substrate surface. This article established different models to elaborate the variation of radiation performance of the quasi-Yagi array under its miniaturized process and carried out detailed quantizing analyses for the decoupling effect.

\section{Quasi-Yagi Antenna Array Design}

The operation frequency band of the communication based train control system is $2.4 \sim 2.48 \mathrm{GHz}$ in rail traffic, and the band is also applied in the wireless local area network (WLAN). This paper designs a quasi-Yagi antenna array with operation frequency $(f)$ of $2.45 \mathrm{GHz}$. The array is fabricated on the substrate with relative permittivity $\left(\varepsilon_{r}\right)$ of 3.3 , and the equivalent wavelength of electromagnetic waves in the substrate $\left(\lambda_{e}\right)$ satisfies the following:

$$
\lambda_{e}=\frac{c}{f \sqrt{\varepsilon_{e}}}=74 \mathrm{~mm},
$$

where $\varepsilon_{e}$ is the effective permittivity of $\varepsilon_{r}$.

The geometry of the quasi-Yagi array structure of different balun compound modes is shown in Figure 1. The wilkinson power divider is used to split the input signal power into two equal power outputs and connected two quasi-Yagi elements as a dualistic antenna array. The thickness of the dielectric substrate is $1 \mathrm{~mm}$, and the exciter, director, balun, and power divider are printed on the front of the substrate, while reflector is printed on the back.

Based on the finite element method, the proposed antenna array is calculated. The array is excited by a side signal through the port as shown in Figure 1.

The S11 of the reference antenna array is simulated as shown in Figure 2. At $2.45 \mathrm{GHz}$, the $\mathrm{S} 11$ of the inverse balun array is $-11.76 \mathrm{~dB}$, and that of the directional balun array is $-11.45 \mathrm{~dB}$.

The $3 \mathrm{D}$ gain simulated results of the antenna array are shown in Figure 3. The inverse balun array forms two symmetrical beams with a maximum gain of $11.15 \mathrm{dBi}$, and the directional balun array forms a single beam with a maximum gain of $13.08 \mathrm{dBi}$.

The above simulation results indicate that the reference antenna array could ensure good radiation performance in the frequency band of $2.4 \sim 2.48 \mathrm{GHz}$.

\section{Characterization of SRR}

To illustrate the decoupling principle of split-ring resonators (SRRs) considered in this work, a model of the SRR unit cell is established as shown in Figure 4.

The SRR consists of a concentric circle ring with an outer radius $(R)$ of $7 \mathrm{~mm}$ and a split gap $(g)$ of $0.5 \mathrm{~mm}$, and the strip width $(w)$ of the ring is $1 \mathrm{~mm}$. The material of SRR is copper with a thickness of $0.035 \mathrm{~mm}$. The relative permittivity of the dielectric substrate is 3.3 with the thickness of $1 \mathrm{~mm}$. The period of the SRR unit cell is $21 \mathrm{~mm}$.

When an $x$-polarized incident wave (the magnetic field direction is along the $y$-axis direction) propagates along the $-z$-axis and passes through the SRR unit cell, a resonance point could be generated by the inductance and capacitance of SRR. At this resonance frequency point, the SRR unit cell would display a single-negative magnetic feature to suppress the propagation of the $x$-polarized incident wave. The scattering parameters and the equivalent electromagnetic parameters of SRR are simulated as shown in Figures 5 and 6 , respectively.

Figure 5 shows the $x$-polarized incident wave is almost completely reflected at the resonance close to $2.5 \mathrm{GHz}$. Figure 6 shows the equivalent magnetic permeability is negative and the equivalent permittivity is positive at the resonance point. The simulated results mean SRR could be regarded as the negative magnetic metamaterials. Due to the fact that the coupling magnetic field of the proposed quasi-Yagi antenna array passes through the SRR array perpendicularly, its mutual coupling could be suppressed.

The electromagnetic performance of SRR with different parameters is discussed as follows.

3.1. Different Radius (R). The equivalent permittivity and permeability of SRR with different $R$ are simulated, respectively, with the values of $7 \mathrm{~mm}, 7.7 \mathrm{~mm}$, and $8.4 \mathrm{~mm}$ and are shown in Figure 6.

Figure 6 shows that with the increase of $R$, the resonance point with a single-negative magnetic feature moves to low frequency.

3.2. Different Split Gap (g). The equivalent permittivity and permeability of SRR with different $g$ are simulated, respectively, $g$ with the values of $0.5 \mathrm{~mm}, 1.0 \mathrm{~mm}$, and $1.5 \mathrm{~mm}$ and are shown in Figure 7.

Figure 7 shows that with the increase of $g$, the resonance point with a single-negative magnetic feature moves to high frequency.

3.3. Different Strip Width $(w)$. The equivalent permittivity and permeability of SRR with different $w$ are simulated, $w$ with the values of $0.6 \mathrm{~mm}, 1.0 \mathrm{~mm}$, and $1.4 \mathrm{~mm}$ and are shown in Figure 8.

Figure 8 shows that with the increase of $w$, the resonance point with a single-negative magnetic feature moves to high frequency.

It can be seen from Figures 6-8 that the resonant frequency with a single-negative magnetic feature of SRR can be adjusted by changing its parameters.

\section{Miniaturized Quasi-Yagi Array Design}

In order to obtain a better performance, the size and arrangement of SRRs are constructed by optimizing design, and the proposed quasi-Yagi antenna arrays with different balun modes are established as shown in Figure 9. The SRRs are embedded on both sides of the dielectric substrate surface with the antenna element spacing of $30 \mathrm{~mm}$ and $40 \mathrm{~mm}$, respectively. 


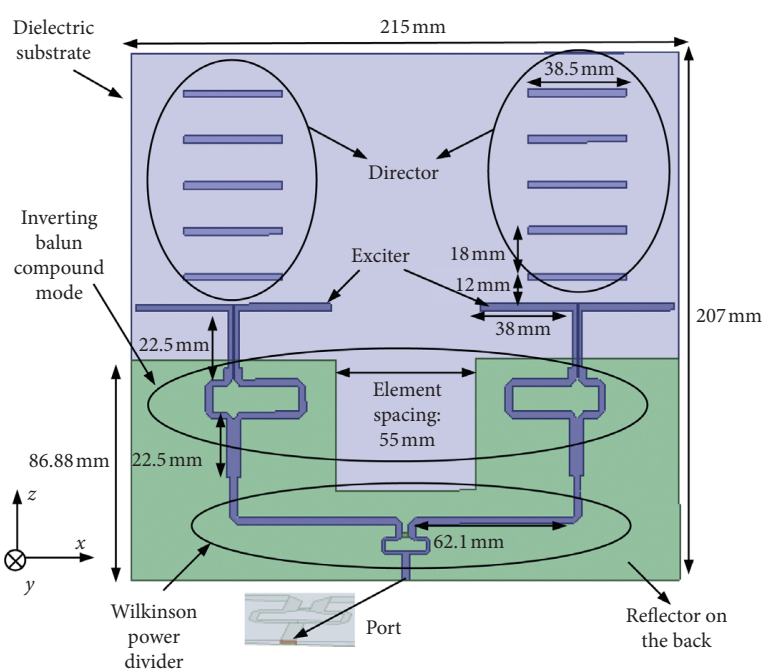

(a)

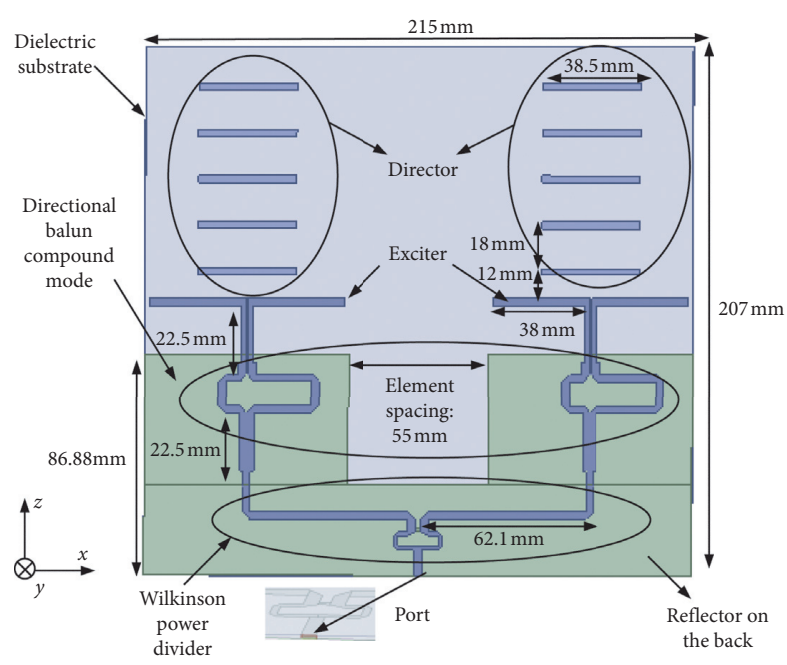

(b)

Figure 1: Reference antenna array structure. (a) Inverse balun. (b) Directional balun.

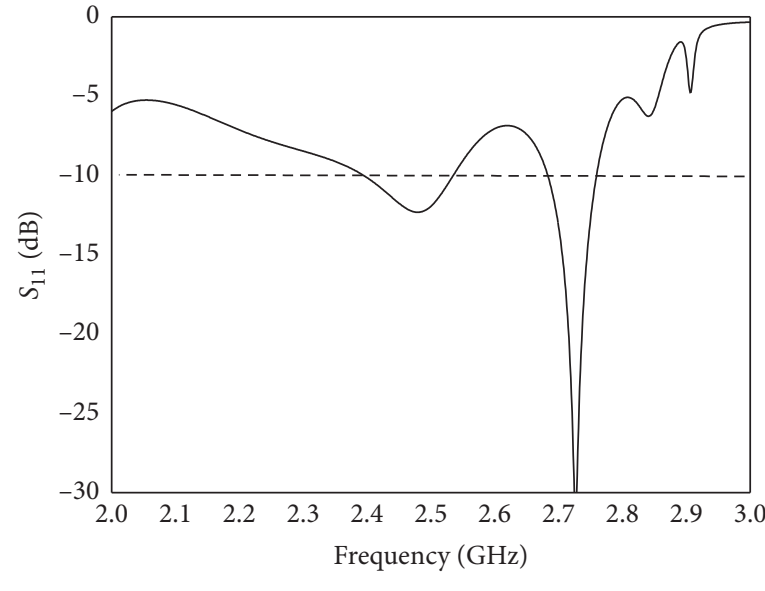

— Without SRRs

(a)

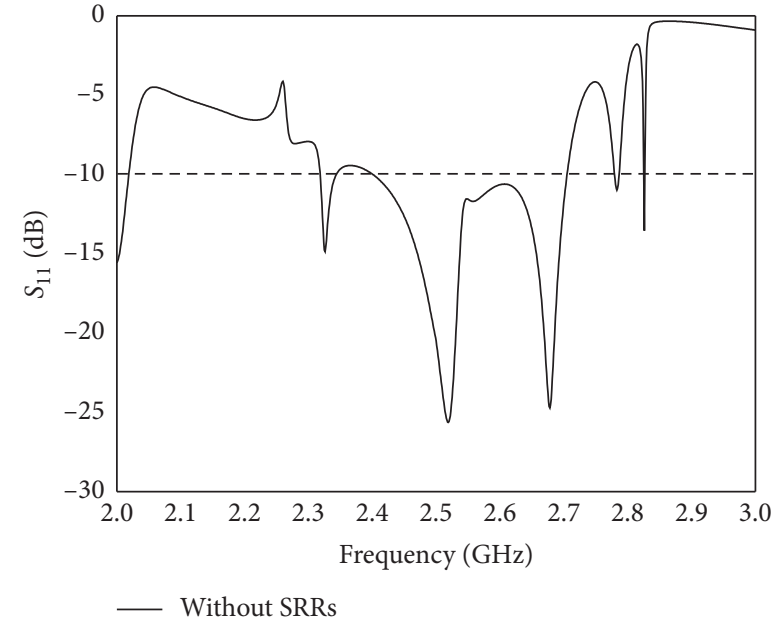

(b)

FIGURE 2: S11 of reference antenna array. (a) Inverse balun. (b) Directional balun.

The variation of antenna array radiation performance under the process of reducing the elements spacing to $40 \mathrm{~mm}$ (decrease of $0.2 \lambda_{e}$ ) and $30 \mathrm{~mm}$ (decrease of $0.34 \lambda_{e}$ ) is compared, respectively.

4.1. The Simulation of Quasi-Yagi Antenna Array with Element Spacing Decrease $\left(0.2 \lambda_{e}\right)$. The S11 comparison of the antenna array with SRRs and without SRRs is shown in Figure 10.

At $2.45 \mathrm{GHz}$, it can be seen from Figure 11(a) that the S11 of the inverse balun quasi-Yagi antenna array with SRRs is $-14.46 \mathrm{~dB}$, and the array without SRRs is $-9.14 \mathrm{~dB}$. It can be seen from Figure 11(b) that the S11 of the directional balun quasi-Yagi antenna array with SRRs is $-12.65 \mathrm{~dB}$, and the array without SRRs is $-2.71 \mathrm{~dB}$. Therefore, the SRRs could further reduce S11 of the quasi-Yagi antenna array.

The 3D gain of the antenna array with SRRs and the array without SRRs is compared, respectively, as shown in Figure 11.

It can be seen from Figures 11(a) and 11(b) that the 3D gain of the inverse balun quasi-Yagi antenna array with SRRs is $13.32 \mathrm{dBi}$ and $2 \mathrm{dBi}$ more than that of the array without SRRs. It can be seen from Figures 11(c) and 11(d) that the 3D gain of the directional balun quasi-Yagi antenna array with SRRs is $12.96 \mathrm{dBi}$, but the array without SRRs appeared with the serious distortion.

As the spacing of the elements decreases, the antenna array without SRRs begins to deteriorate, and the degree of wave beam separation is worsened. Adding SRRs not only 


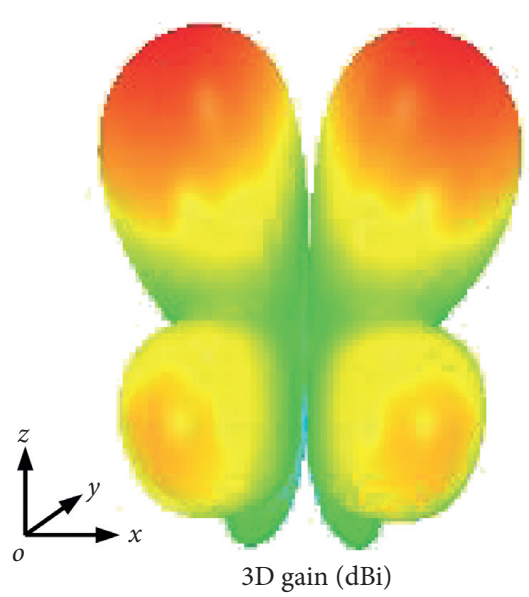

(a)

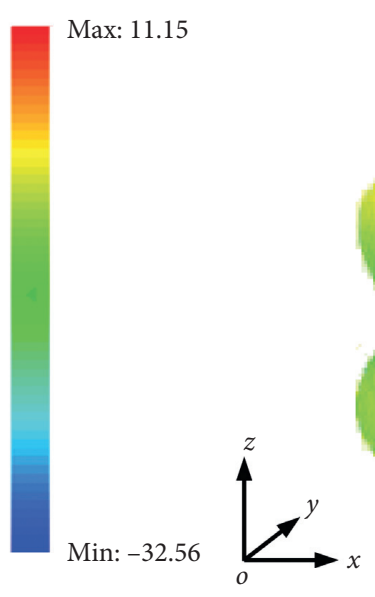

FIGURE 3: 3D gain of the reference antenna array. (a) Inverse balun. (b) Directional balun.

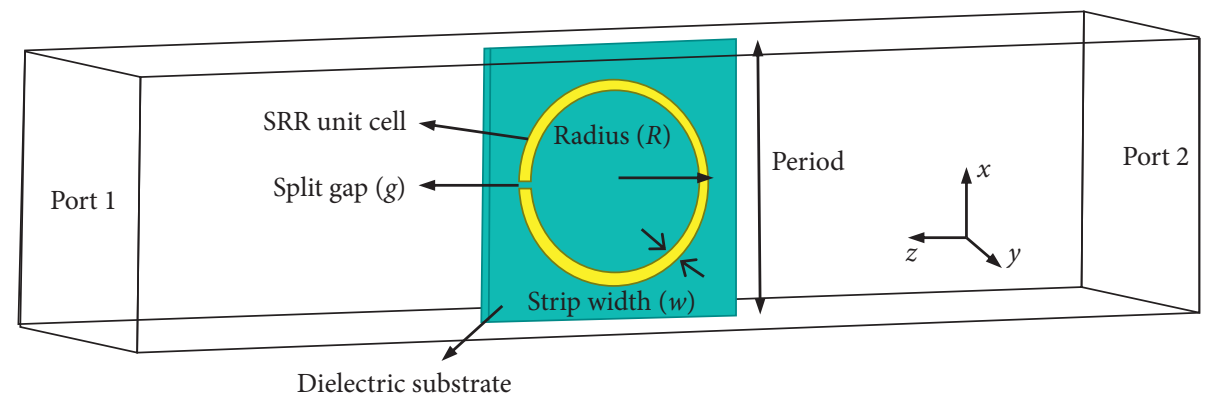

FIGURE 4: Waveguide structure used for characterization of the SRR unit cell ( $E$-field points the $x$-direction, $H$-field is in the $y$-direction, and the propagation is the $z$-direction).

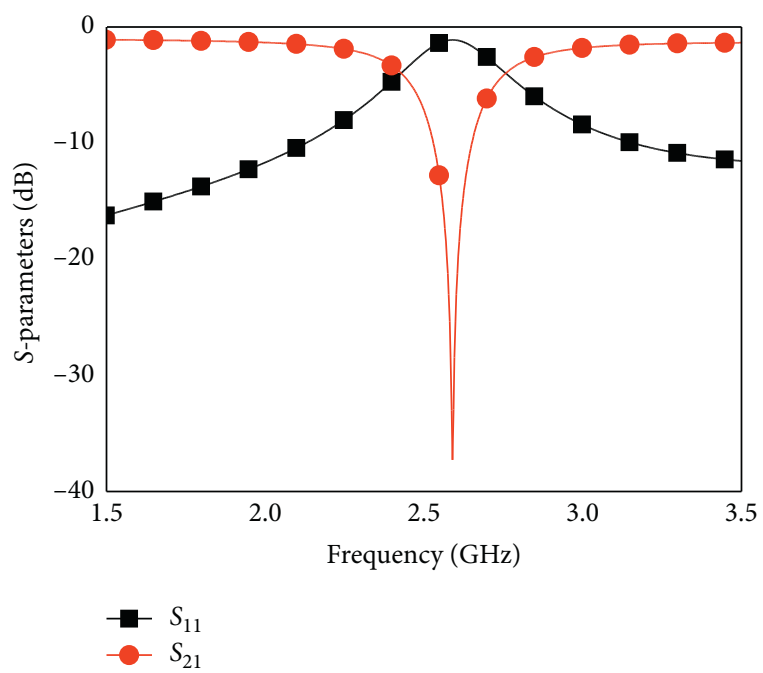

FIGURE 5: Scattering parameters of a SRR unit cell.

effectively overcomes the distortion of the antenna array but also separates beam energy effectively and enhances gain by $1.05 \sim 1.13$ times.

In order to further analyse the decoupling effect, the electric field intensity distribution of the antenna array with
SRRs and the array without SRRs is compared, as shown in Figure 12 .

The induced current generated on the five directors would intensify electric field superposition, which increases the mutual coupling effected. Because of the resonance effect, adding SRRs could effectively balance the electric field strength on the two elements.

4.2. The Simulation of Quasi-Yagi Antenna Array with Element Spacing Decrease $\left(0.34 \lambda_{e}\right)$. The $S 11$ comparison of the antenna array with SRRs and without SRRs is shown in Figure 13. At $2.45 \mathrm{GHz}$, it can be seen from Figure 13(a) that the S11 of the inverse balun quasi-Yagi antenna array with SRRs is $-13.11 \mathrm{~dB}$, and that of the array without SRRs is $-8.81 \mathrm{~dB}$. It can be seen from Figure 13(b) that the $S 11$ of the directional balun quasi-Yagi antenna array with SRRs is $-15.91 \mathrm{~dB}$, and that of the array without SRRs is $-13.52 \mathrm{~dB}$.

The 3D gain of the antenna array with SRRs and the array without SRRs is compared, respectively, as shown in Figure 14.

It can be seen from Figure 14(a) that the 3D gains of the inverse balun quasi-Yagi antenna array with SRRs is $11.77 \mathrm{dBi}$ and $2.02 \mathrm{dBi}$ more than that of the array without SRRs. It can be seen from Figure 14(b) that the 3D gain of the 


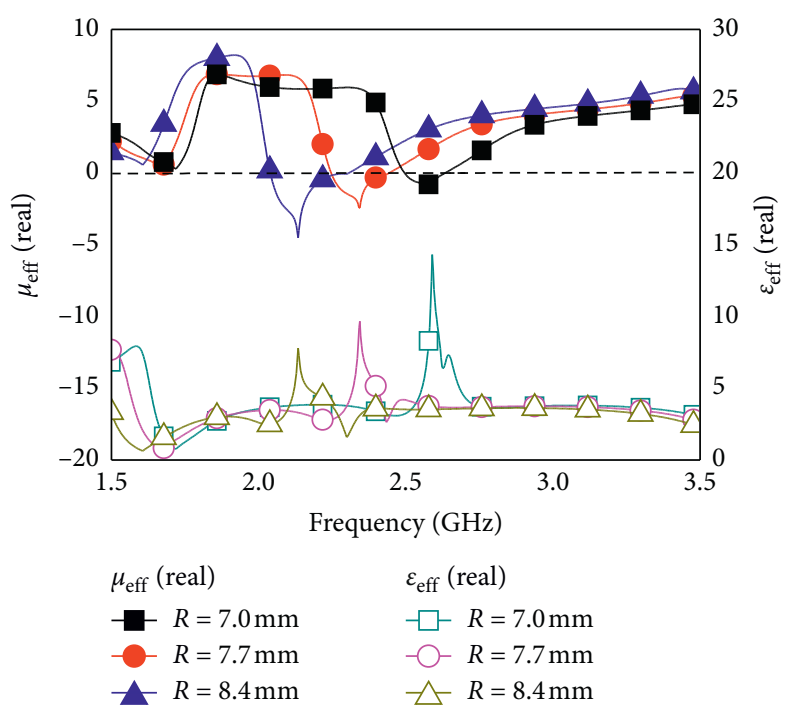

FIGURE 6: Effective response of SRR with different $R$.

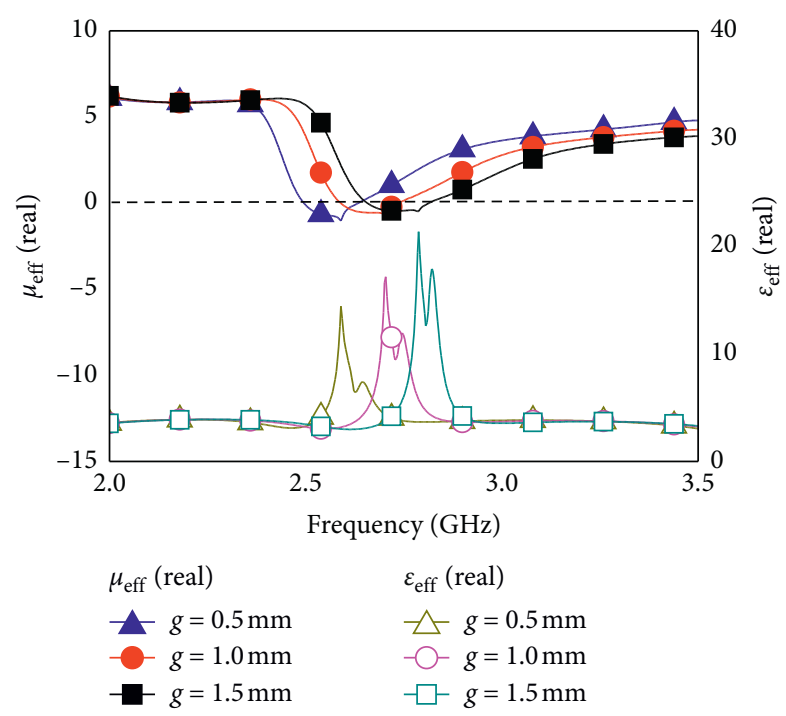

FIGURE 7: Effective response of SRR with different $g$.

directional balun quasi-Yagi antenna array with SRRs is $12.14 \mathrm{dBi}$ and $5.04 \mathrm{dBi}$ more than that of the array without SRRs.

The electric field intensity distribution of the antenna array with SRRs and the array without SRRs is compared, as shown in Figure 15.

As the spacing of the elements is further reduced, the mutual coupling effect is further aggravated correspondingly. The SRRs could form a more symmetric distribution of the antenna array to reduce the mutual coupling and ensure that the radiation performance of the antenna array is not affected.

The radiation performance of the antenna array with decreased element spacing to the reference antenna array at $2.45 \mathrm{GHz}$ is compared, as shown in Table 1.

Table 1 shows that in the process of reducing the array element spacing, the S11 of the antenna array with SRRs is

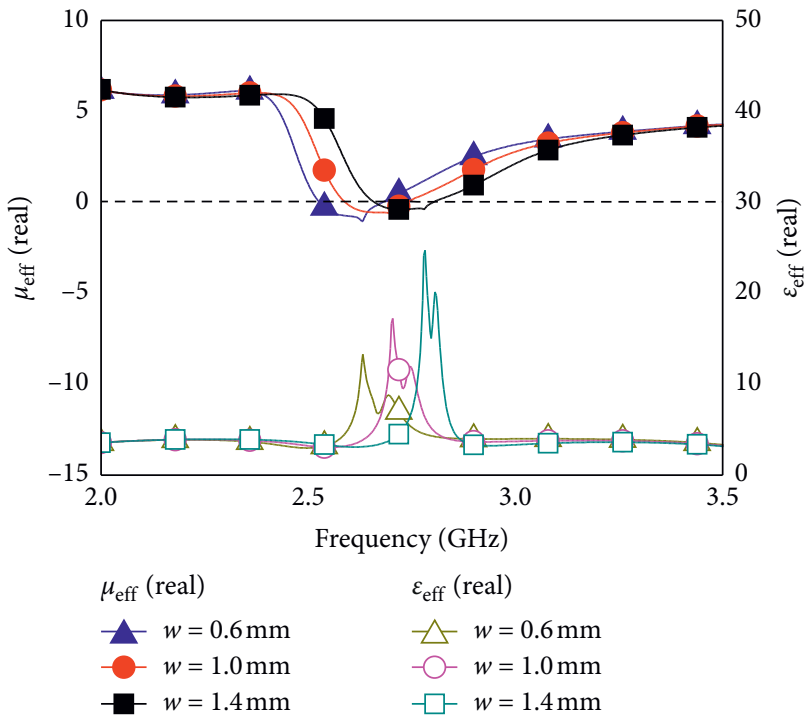

FIgURE 8: Effective response of SRR with different $w$.

much lower than $-10 \mathrm{~dB}$, which achieves the engineering requirements. It indicates that the antenna array with SRRs could reduce the mutual coupling and get better matching characteristics.

\section{Antenna Array Fabrication and Its Measurement Results}

The manufactured antenna array prototypes with different element spacing are shown in Figure 16. The fabricated dielectric substrate has same relative permittivity and thickness as the simulation model. The reference array is shown in Figures 16(a) and 16(b), and the proposed antenna array with SRRs is observed in Figures 16(c) and 16(d). 


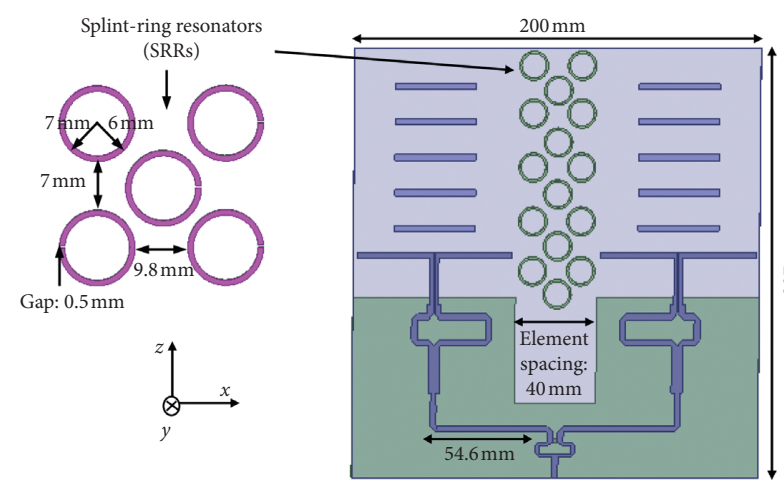

(a)

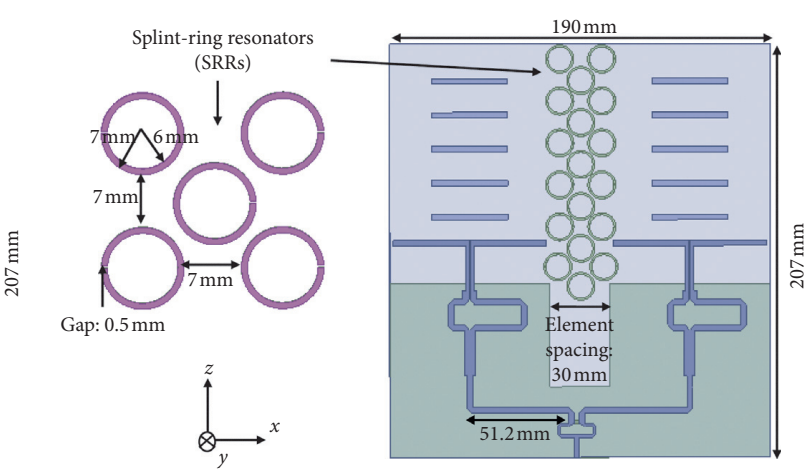

(b)

FIGURE 9: Quasi-Yagi antenna array with SRRs. (a) Inverse balun. (b) Directional balun.

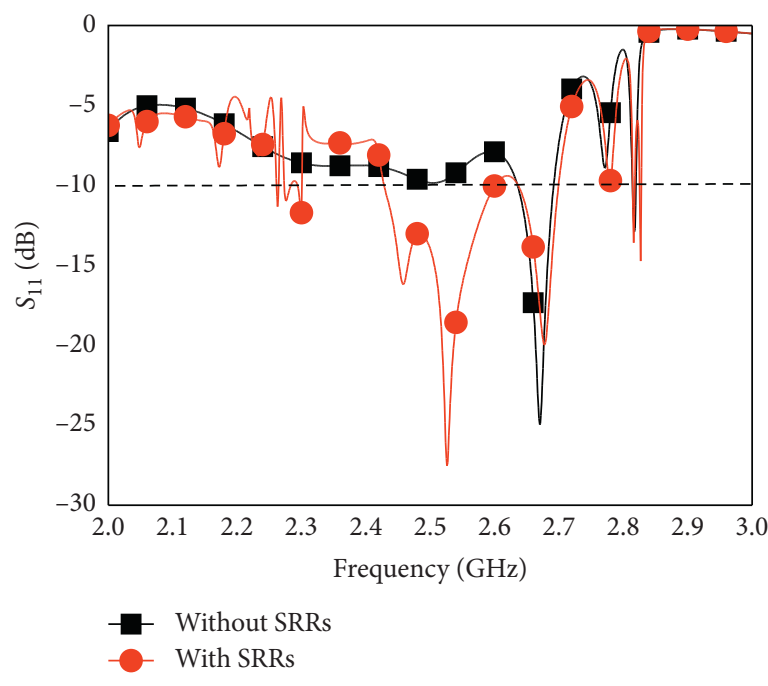

(a)

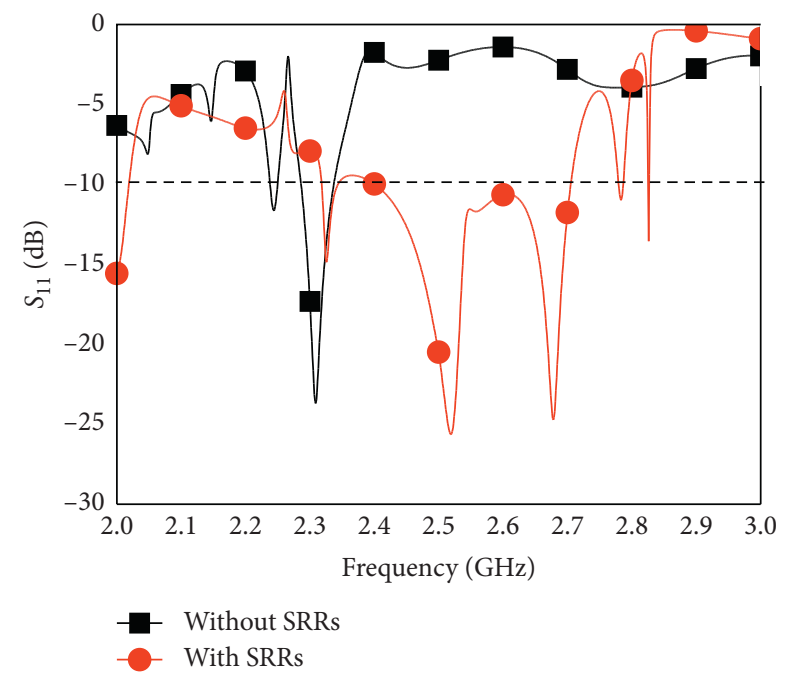

(b)

FIgURe 10: Comparison of the S11 $\left(0.2 \lambda_{e}\right)$. (a) Inverse balun. (b) Directional balun.

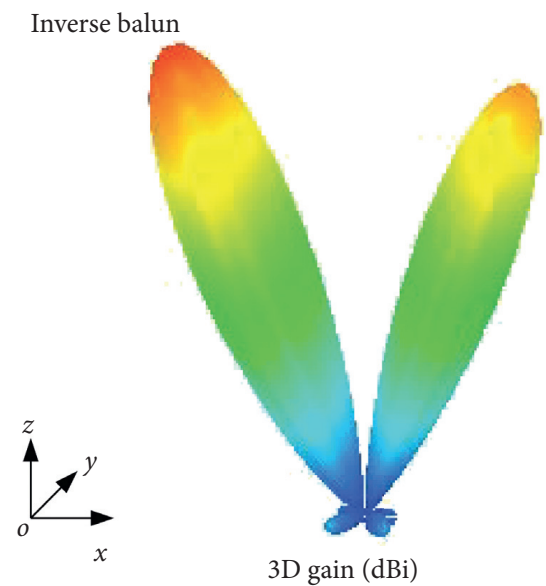

(a)

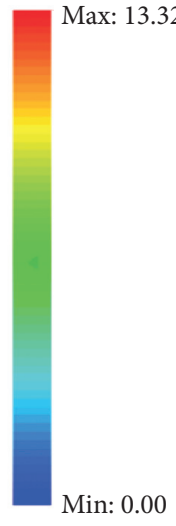

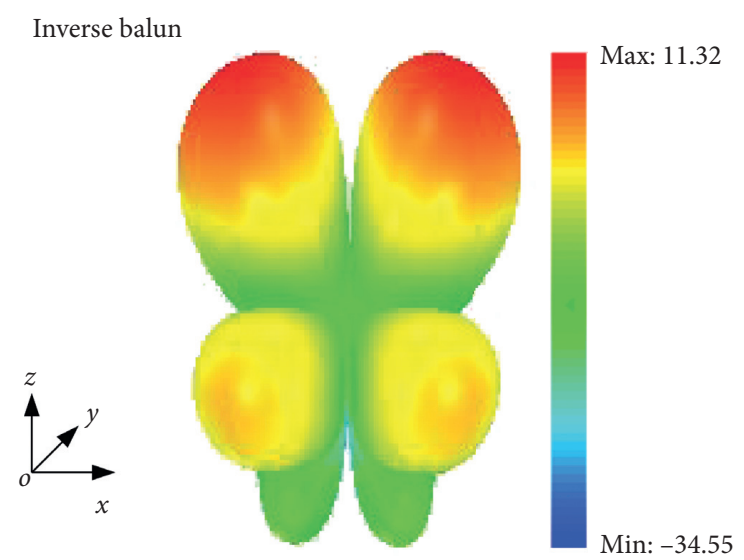

3D gain $(\mathrm{dBi})$

(b)

Figure 11: Continued. 


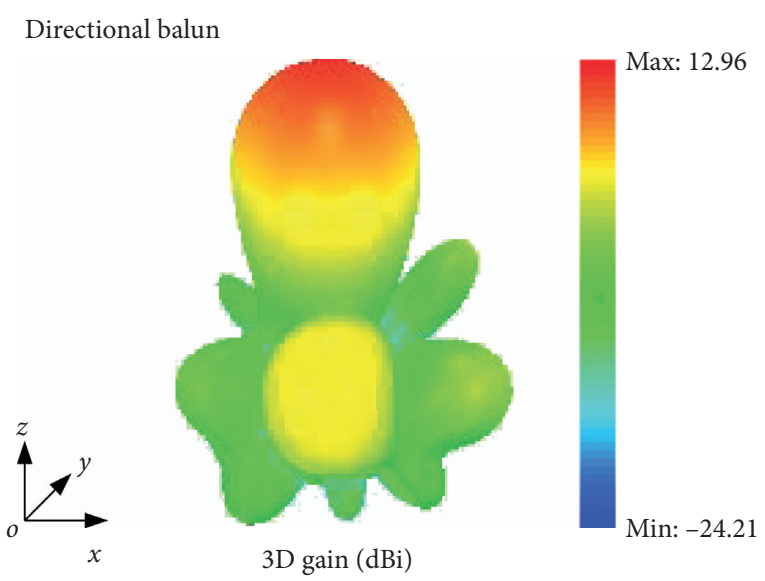

(c)

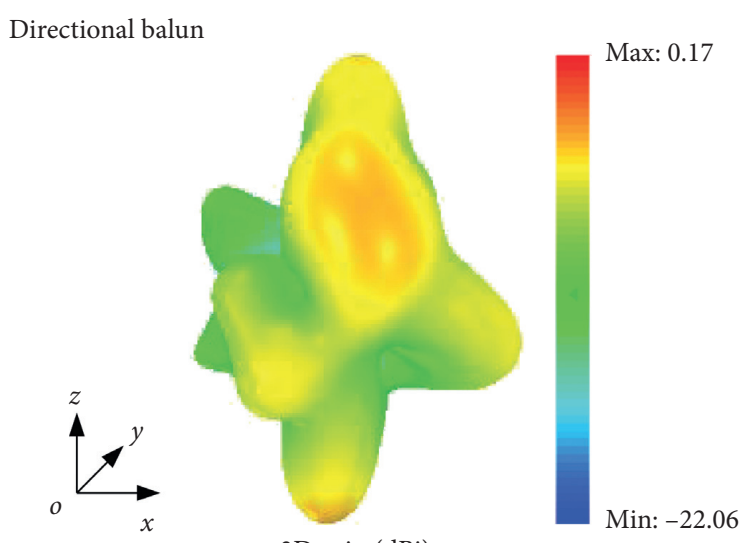

$3 \mathrm{D}$ gain $(\mathrm{dBi})$

(d)

FIgURE 11: Comparison of 3D gain $\left(0.2 \lambda_{e}\right)$. (a) Array with SRRs. (b) Array without SRRs. (c) Array with SRRs. (d) Array without SRRs.
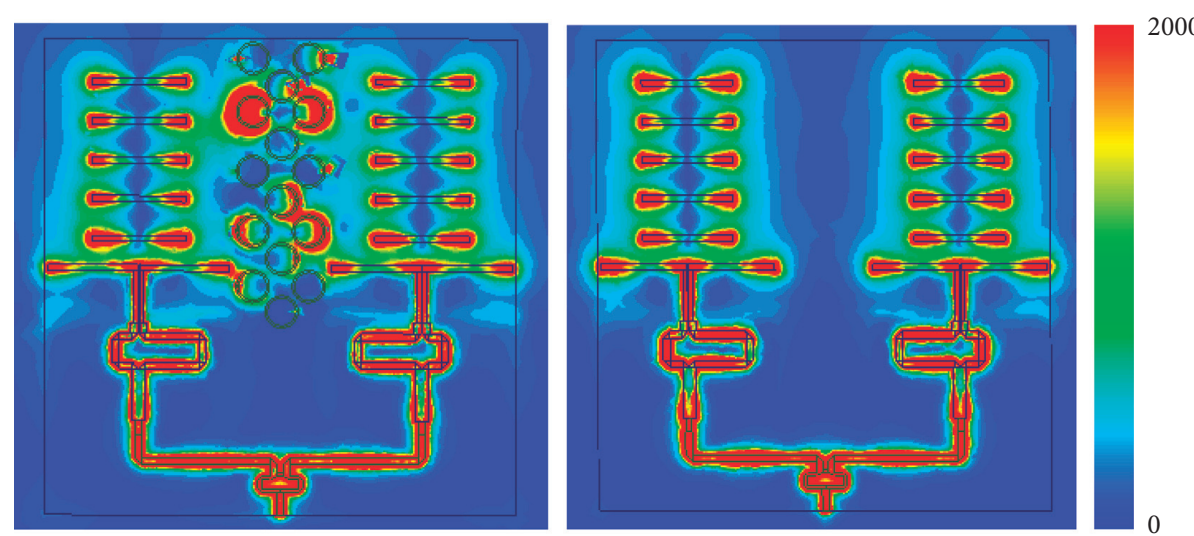

$|E|(\mathrm{V} / \mathrm{m})$

(a)
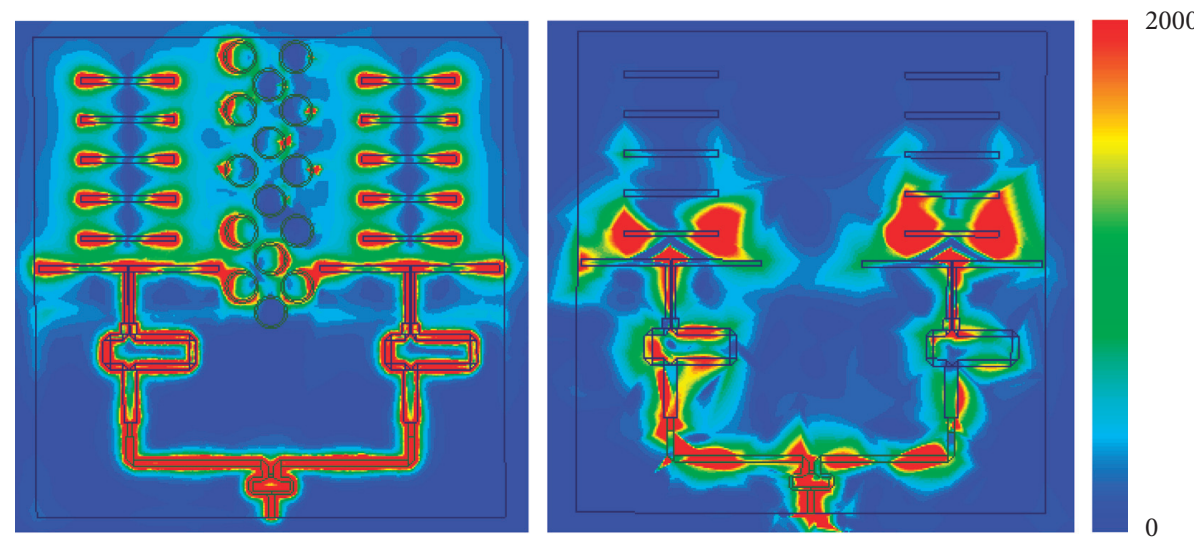

$|E|(\mathrm{V} / \mathrm{m})$

(b)

Figure 12: Electric field intensity distribution of the antenna array $\left(0.2 \lambda_{e}\right)$. (a) Inverse balun. (b) Directional balun.

The fabricated design is measured by using a Fieldfox N9917A microwave analyzer, and the measurement results of S11 are shown in Figure 17.

It can be seen that the error between the simulation value and the measured value is less than $1.2 \%$. At the
$2.45 \mathrm{GHz}$, the $\mathrm{S} 11$ measurement value of the array with SRRs is $-13.49 \mathrm{~dB}$, which is $-2.5 \mathrm{~dB}$ better than the reference array.

The measurement results of the radiation pattern are shown in Figure 13. 


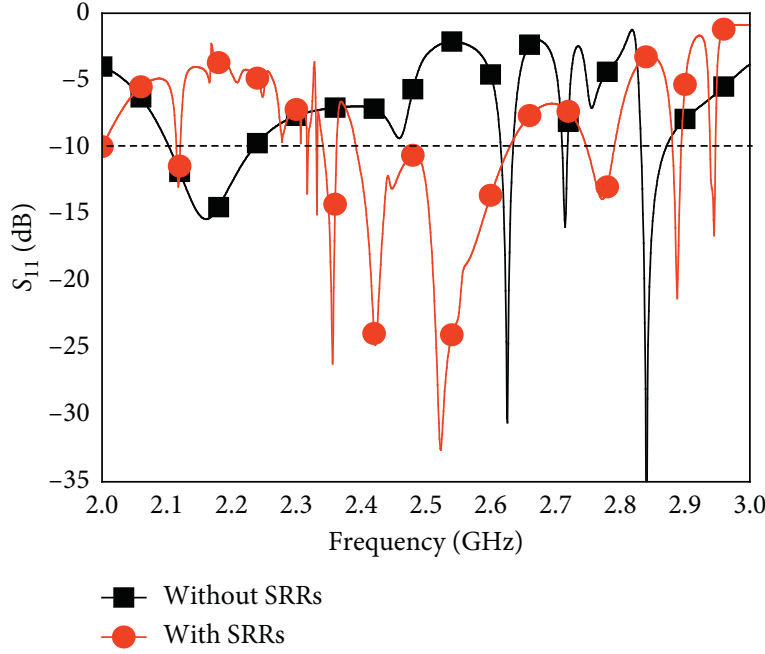

(a)

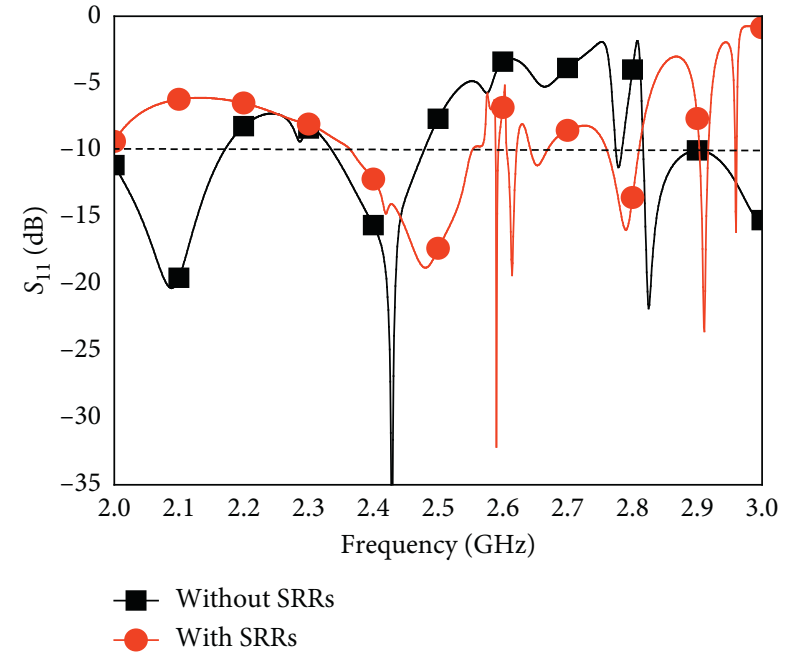

(b)

FIGURE 13: Comparison of S11 of the array $\left(0.34 \lambda_{e}\right)$. (a) Inverse balun. (b) Directional balun.

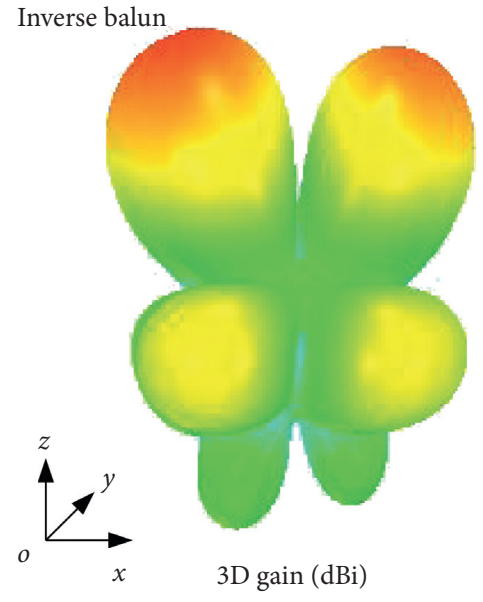

(a)

Directional balun

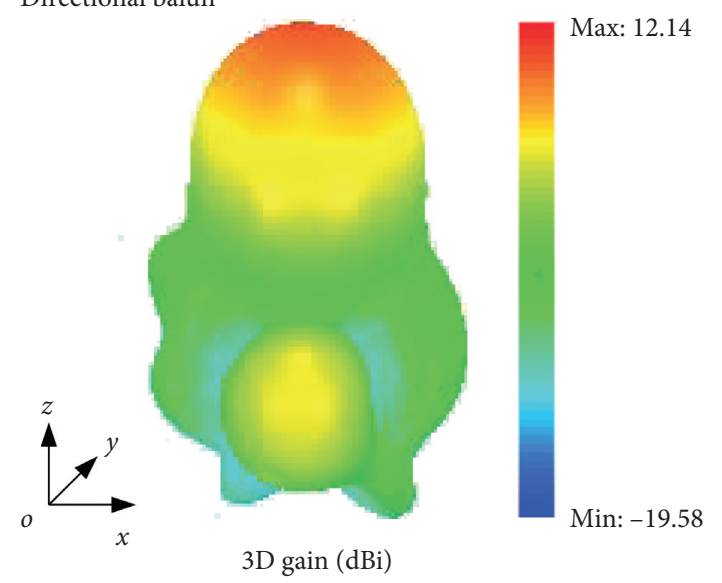

(c)

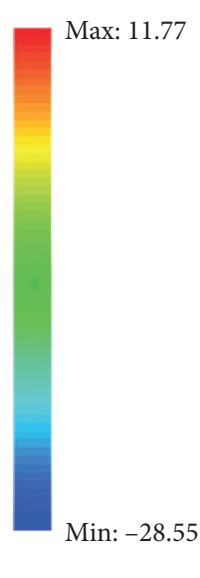

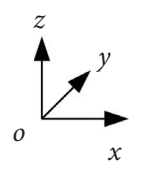

(b)

Directional balun

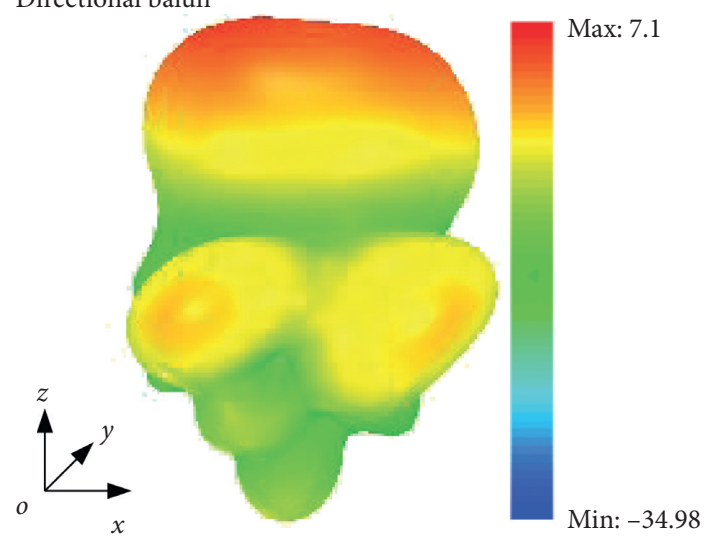

3D gain $(\mathrm{dBi})$

(d)

FIgURE 14: Comparison of the 3D gain $\left(0.34 \lambda_{e}\right)$. (a) Array with SRRs. (b) Array without SRRs. (c) Array with SRRs. (d) Array without SRRs. 


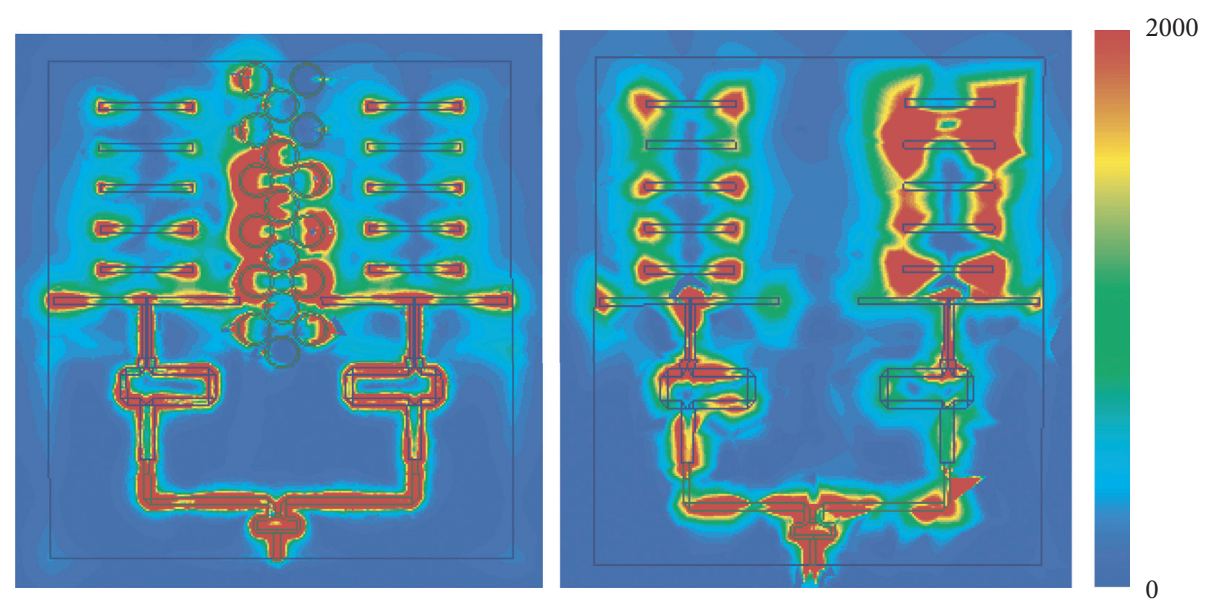

$|E|(\mathrm{V} / \mathrm{m})$

(a)
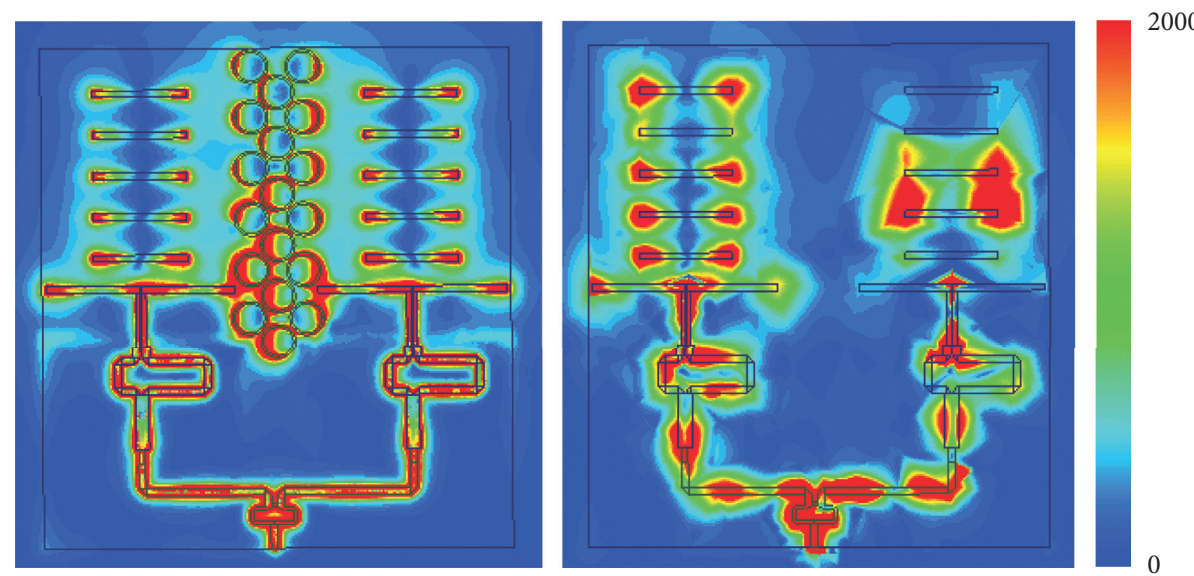

$|E|(\mathrm{V} / \mathrm{m})$

(b)

FIgURE 15: Electric field intensity distribution of the antenna array $\left(0.34 \lambda_{e}\right)$. (a) Inverse balun. (b) Directional balun.

TABLE 1: Comparison of the radiation performance of the proposed antenna array with SRRs and without SRRs.

\begin{tabular}{lcccc}
\hline & Element spacing & $30 \mathrm{~mm}$ (SRRs/without SRRs) & $40 \mathrm{~mm}$ (SRRs/without SRRs) & $55 \mathrm{~mm}$ (without SRRs) \\
\hline \multirow{2}{*}{ Inverse balun } & $\mathrm{S} 11 \mathrm{~dB})$ & $-13.11 /-8.81$ & $-14.46 /-9.14$ & -11.76 \\
& Maximum gain (dBi) & $11.77 / 9.75$ & $13.32 / 11.32$ & 11.15 \\
\hline \multirow{2}{*}{ Directional balun } & $\mathrm{S} 11(\mathrm{~dB})$ & $-15.91 /-13.52$ & $-12.65 /-2.71$ & -11.45 \\
& Maximum gain (dBi) & $12.14 / 7.1$ & $12.96 / 0.17$ & 13.08 \\
\hline
\end{tabular}

It can be observed from Figure 18(a) that the gain of the antenna array at $-22^{\circ}$ is $11.1 \mathrm{dBi}$ and at $20^{\circ}$ is $12 \mathrm{dBi}$; from Figure $18(\mathrm{~b})$, the gain of the antenna array at $-22^{\circ}$ is $10 \mathrm{dBi}$ and at $22^{\circ}$ is $12 \mathrm{dBi}$. The compared results indicate a very decent maximum gain matching achieved in the direction of the main lobe. The slight variation in the side lobe is attributed to inevitable error in the manufacturing process and different tangent loss of the substrate.

\section{Conclusion}

In this paper, the mutual coupling of the quasi-Yagi antenna array is investigated at $2.45 \mathrm{GHz}$. The effect of SRRs on the radiation performance of the quasi-Yagi antenna array during the element spacing reduced process is simulated. The simulation results indicate that the SRRs could suppress mutual coupling effectively and guarantee high gain. The measurement results of 


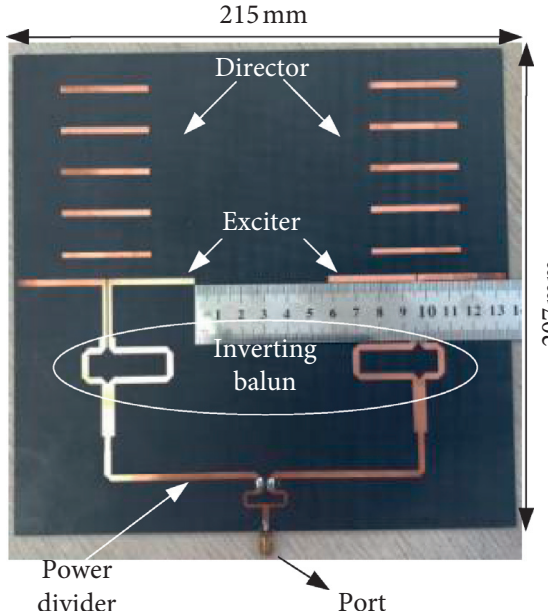

(a)

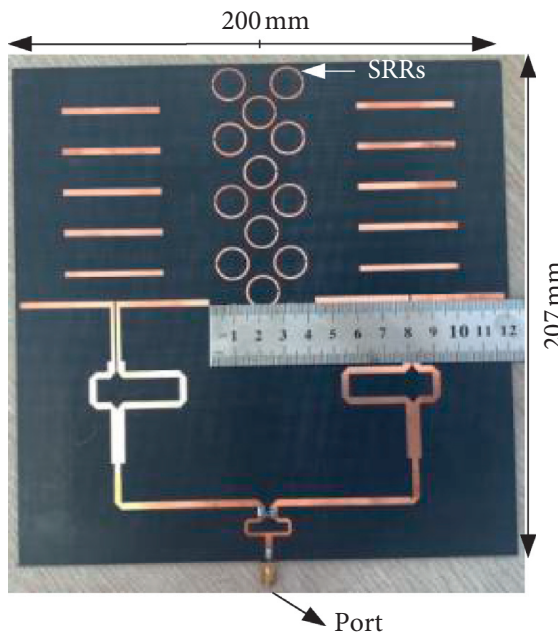

(c)

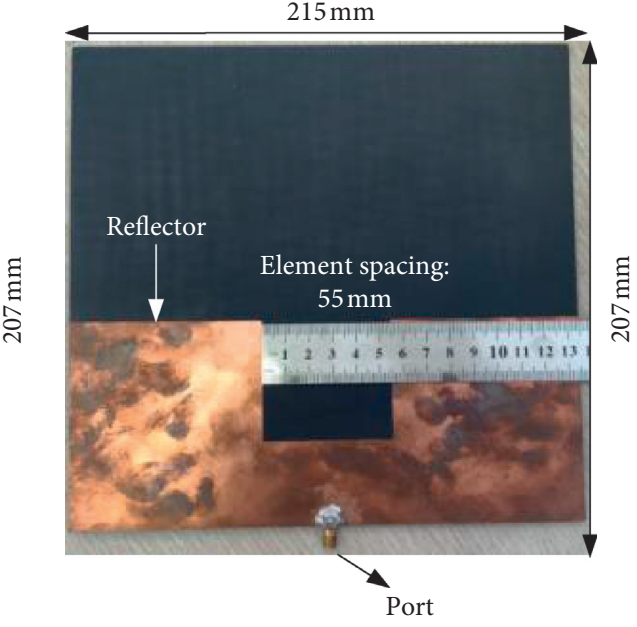

(b)

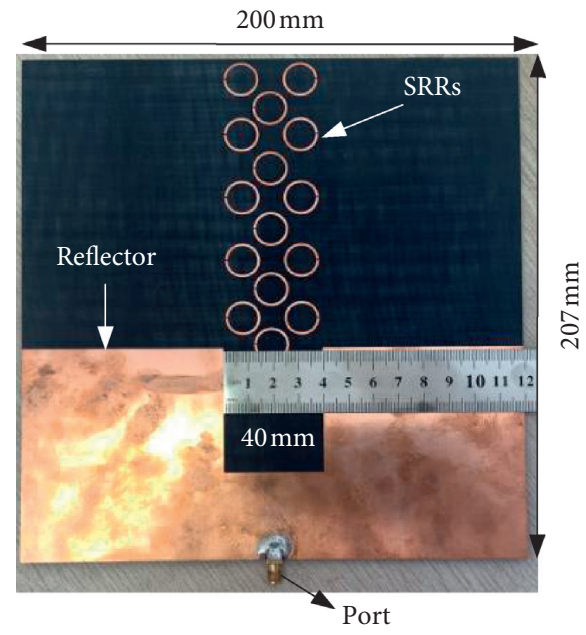

(d)

Figure 16: Prototype of the quasi-Yagi antenna array. (a) Top view of the reference array $(55 \mathrm{~mm})$. (b) Bottom view of the reference array $(55 \mathrm{~mm})$. (c) Top view of the array with SRRs $(40 \mathrm{~mm})$. (d) Bottom view of the array with SRRs $(40 \mathrm{~mm})$.

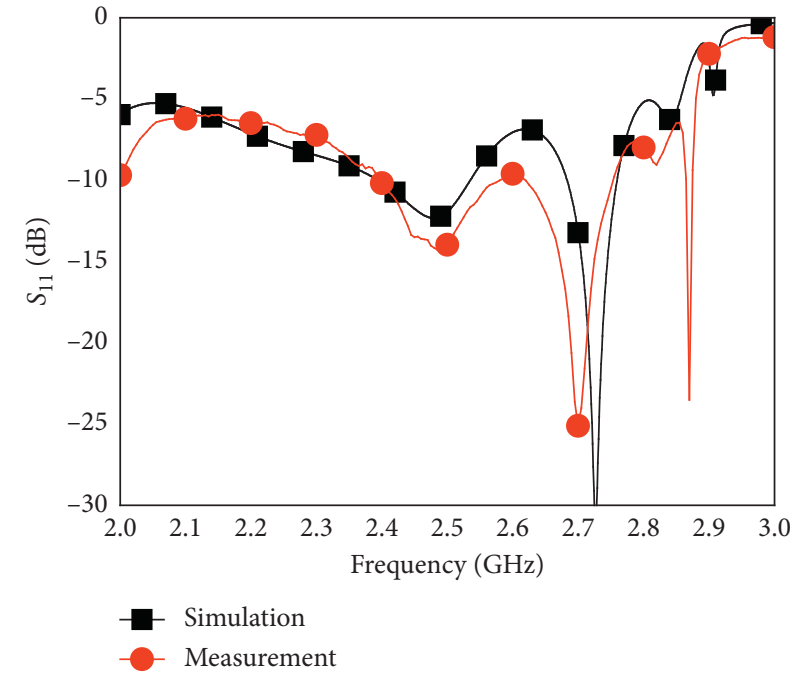

(a)

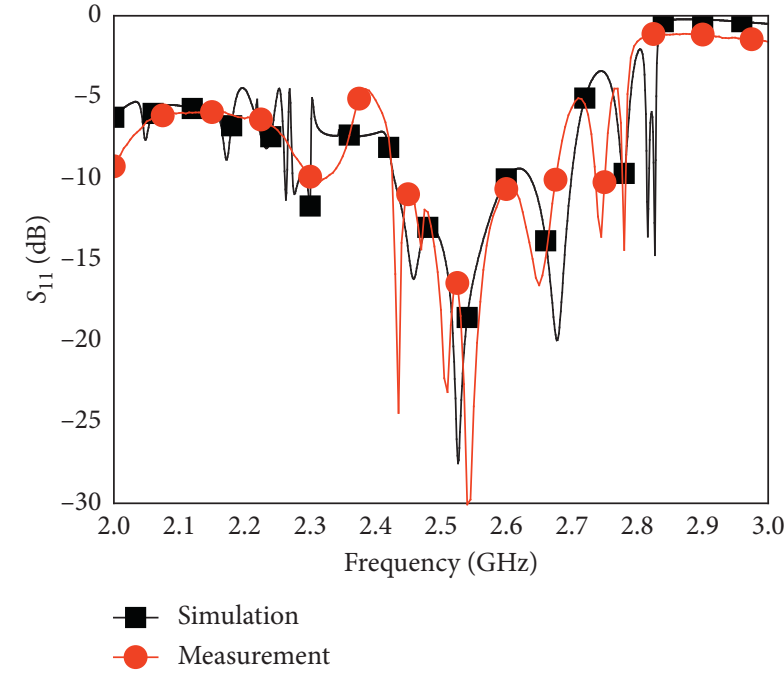

(b)

FIgURE 17: Comparison of the S11 (prototype). (a) Antenna array without SRRs. (b) Antenna array with SRRs. 


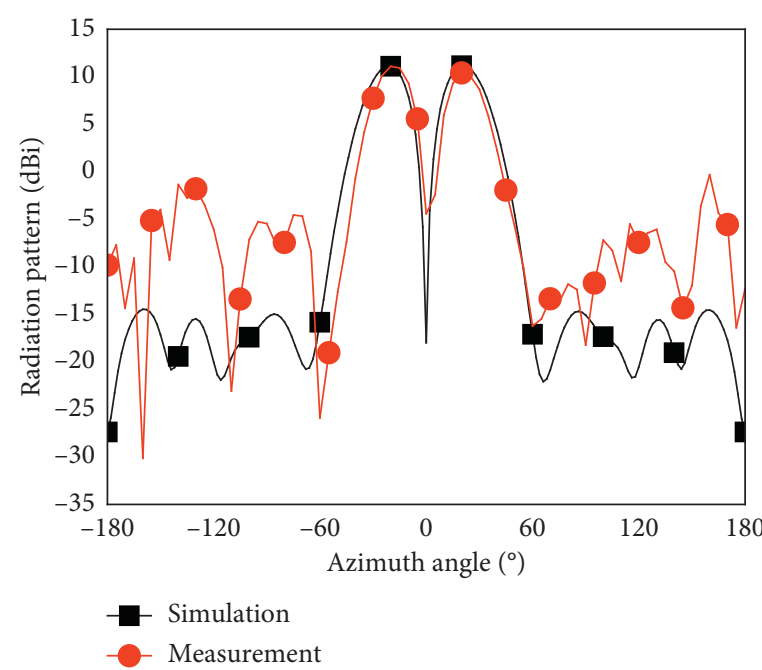

(a)

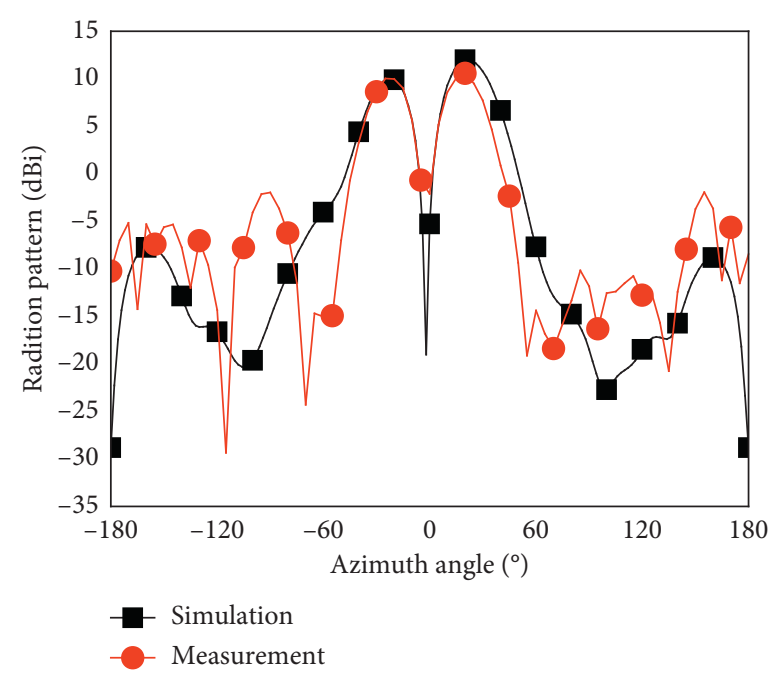

(b)

FIGURE 18: Comparison of the radiation pattern (prototype)

(a) Antenna arrays without SRRs. (b) Antenna arrays with SRRs.

miniaturized quasi-Yagi antenna array fabrication show a better radiation performance compared with the reference array.

The work is useful for quasi-Yagi antenna miniaturization and could obtain better application in transportation and WPT. The proposed SRR structure needs to be further optimized to adapt different antenna arrays.

\section{Data Availability}

The data used to support the findings of this study are included within the article.

\section{Conflicts of Interest}

The authors declare that they have no conflicts of interest.

\section{Acknowledgments}

This research was financially supported by the National Natural Science Foundation of China (61701208, 51567015 and 51867014), the Department of Education of Gansu Province (Grant nos. 2018D-08), and the Tianyou Youth Talent Lift Program of Lanzhou Jiaotong University (1520260111).

\section{References}

[1] J. Moreno, L. de Haro, C. Rodriguez, L. Cuellar, and J. M. Riera, "Keyhole estimation of an MIMO-OFDM trainto-wayside communication system on subway tunnels," IEEE Antennas and Wireless Propagation Letters, vol. 14, pp. 88-91, 2015.

[2] R. He, Z. Zhong, K. Guan, B. Chen et al., "Propagation channel measurements and analysis at $2.4 \mathrm{GHz}$ in subway tunnels," IET Microwaves, Antennas \& Propagation, vol. 7, no. 11, pp. 934-941, 2013.

[3] X. X. Wang, L. J. Liu, T. Tang, and W. Sun, "Enhancing communication-based train control systems through train-to- train communications," IEEE Transactions Intelligent Transportation System, vol. 20, no. 4, pp. 1-18, 2019.

[4] P. Soboll, V. Wienstroer, and R. Kronberger, "Stacked YagiUda array for $2.45-\mathrm{GHz}$ wireless energy harvesting," IEEE Microwave Magazine, vol. 16, no. 1, pp. 67-73, 2015.

[5] D. Rupam and Y. Hyoung, "Wireless power transfer to a pacemaker by using metamaterials and Yagi-Uda antenna concept," in Proceedings of the International Conference on iWAT, pp. 353-354, Seoul, South Korea, March 2015.

[6] B. Zhou, A. Liu, and V. Lau, "Successive localization and beamforming in $5 \mathrm{G}$ mmWave MIMO communication systems," IEEE Transactions on Signal Processing, vol. 67, no. 6, pp. 1620-1635, 2019.

[7] H. R. Khaleel, H. AI-Rizzo, A. Abbosh, and S. Abushamleh, "Printed Yagi-Uda array for mimo systems," in Proceedings of the International Conference on Antennas and Propagation Society International Symposium (APSURSI), pp. 1822-1823, IEEE, Orlando, FL, USA, July 2013.

[8] S. S. Jehangir and M. S. Sharawi, "A wideband sectoral quasiYagi MIMO antenna system with multibeam elements," IEEE Transactions on Antennas and Propagation, vol. 67, no. 3, pp. 1898-1903, 2019.

[9] K. P. Ray and N. M. Ammanagi, "Investigation of mutual coupling effect on the performance of 3-element Yagi-Uda antenna in linear and planar array configuration," AEU-International Journal of Electronics and Communications, vol. 65, no. 6, pp. 510-515, 2011.

[10] G. Shen, Y. Liu, G. Sun, T. Zheng, X. Zhou, and A. Wang, "Suppressing sidelobe level of the planar antenna array in wireless power transmission," IEEE Access, vol. 7, pp. 69586970, 2019.

[11] N. Parhizgar, A. Alighanbari, M.-A. Masnadi-Shirazi, and A. Sheikhi, "Mutual coupling compensation for a practical VHF/UHF Yagi-Uda antenna array," IET Microwaves, Antennas \& Propagation, vol. 7, no. 13, pp. 1072-1083, 2013.

[12] F. Yang and Y. Rahmat-Samii, "Microstrip antennas integrated with electromagnetic band-gap (EBG) structures: a low mutual coupling design for array application," IEEE Transactions on Antennas and Propagation, vol. 51, no. 10, pp. 2936-2946, 2003. 
[13] M. Kim and D. G. Kam, "A wideband and compact EBG structure with a circular defected ground structure," IEEE Transactions on Components, Packaging and Manufacturing Technology, vol. 4, no. 3, pp. 496-503, 2014.

[14] S. Kuzu and N. Akcam, "Array antenna using defected ground structure shaped with fractal form generated by Apollonius circle," IEEE Antennas and Wireless Propagation Letters, vol. 16, pp. 1020-1023, 2017.

[15] J. Banerjee, A. Karmakar, R. Ghatak, and D. R. Poddar, "Compact CPW-fed UWB MIMO antenna with a novel modified minkowski fractal defected ground structure (DGS) for high isolation and triple band-notch characteristic," Journal of Electromagnetic Waves and Applications, vol. 31, no. 15, pp. 1550-1565, 2017.

[16] H. X. Zhang, N. Xu, and L. Y. Huang, "Reducing mutual coupling in microstrip antenna array using metamaterial spiral resonator," IJCSI, vol. 9, no. 3, pp. 51-56, 2012.

[17] L. Zhang, S. Zhang, Z. Song, Y. Liu, L. Ye, and Q. H. Liu, "Adaptive decoupling using tunable metamaterials," IEEE Transactions on Microwave Theory and Techniques, vol. 64, no. 9, pp. 2730-2739, 2016.

[18] D. A. Ketzaki and T. V. Yioultsis, "Metamaterial-based design of planar compact MIMO monopoles," IEEE Transactions on Antennas and Propagation, vol. 61, no. 5, pp. 2758-2766, 2013.

[19] B. Yioultsis and A. Kunte, "Improved design CELC metaresonators for bandwidth improvement and miniaturization of patch antenna," Applied Physics A, vol. 124, no. 12, p. 860, 2018.

[20] M. M. Bait-Suwailam, M. S. Boybay, and O. M. Ramahi, "Electromagnetic coupling reduction in high-profile monopole antennas using single-negative magnetic metamaterials for MIMO Applications," IEEE Transactions on Antennas and Propagation, vol. 58, no. 9, pp. 2894-2902, 2010.

[21] Z. Qamar, L. Riaz, S. A. Khan, M. Chongcheawchamnan, and M. F. Shafique, "Slot combined complementary split ring resonators for mutual coupling suppression in microstrip phased arrays," IET Microwaves, Antennas \& Propagation, vol. 8, no. 15, pp. 1261-1267, 2014.

[22] Q. W. Ye, Y. Liu, H. Lin, M. Li, and H. Yang, "Multi-band metamaterial absorber made of multi-gap, SRRs Structure," Applied Physics A, vol. 107, no. 1, pp. 155-160, 2012.

[23] A. Habshi, J. Nourinia, and C. Ghobadi, "Mutual coupling reduction between very closely spaced patch antennas using low-profile folded split-ring resonators (FSRRs)," IEEE Antennas and Wireless Propagation Letters, vol. 10, pp. 862-865, 2011. 